\title{
Human plasmacytoid dendritic cells acquire phagocytic capacity by TLR9 ligation in the presence of soluble factors produced by renal epithelial cells
}

Running title: GM-CSF enhances phagocytic capacity of pDCs

Jurjen M. Ruben ${ }^{1}$, Gina Stella García-Romo ${ }^{1,3}$, Eytan Breman ${ }^{1,4}$, Sandra van der Kooij ${ }^{1}$, Anke Redeker $^{2}$, Ramon Arens ${ }^{2}$, Cees van Kooten ${ }^{1}$

${ }^{1}$ Department of Nephrology, Leiden University Medical Center, Leiden, The Netherlands ${ }^{2}$ Department of Immunohematology and Blood Transfusion, Leiden University Medical Center, Leiden, The Netherlands

${ }^{3}$ Current address: Morphophysiology Unit, Faculty of Iztacala, National Autonomous University of Mexico, Los Reyes Ixtacala, Tlalnepantla, Estado de México, CP, 54090, México. ${ }^{4}$ Current address: Celyad S.A., Rue Edouard Belin, 21435 Mont-Saint-Guibert, Belgium

Correspondence:

kooten@lumc.nl

Cees van Kooten

Department of Nephrology

Leiden University Medical Center, C7-Q

Albinusdreef 2, $2333 \mathrm{ZA}$, Leiden,

The Netherlands 


\begin{abstract}
Plasmacytoid dendritic cells ( $\mathrm{pDCs}$ ) are antigen presenting cells (APCs) specialized in viral recognition through Toll-like receptor (TLR)7 and TLR9, and produce vast amounts of interferon alpha (IFN $\alpha$ ) upon ligation of these TLRs. We have previously demonstrated a strong influx of pDCs in the tubulointerstitium of renal biopsies at time of acute rejection. However, the role of human pDCs in mediating acute or chronic allograft rejection remains elusive. pDCs are thought to have a limited capacity to ingest apoptotic cells (ACs), which is critical for inducing $\mathrm{CD} 4^{+} \mathrm{T}$ cell activation via indirect antigen presentation and subsequent activation of antibody producing B cells. Here we set out to investigate whether the function of pDCs is affected by their presence within the graft. We show that maturation and IFN $\alpha$ production by pDCs was enhanced when cells were activated in the presence of viable HK2 renal epithelial cells. Importantly, soluble factors produced by CMV-infected (primary) epithelial or endothelial cells enhanced pDC activation, and induced their capacity to phagocytose apoptotic cells. Phagocytosis was not induced by free virus or soluble factors from non-infected cells. Activated pDCs showed an enhanced $\mathrm{CD}^{+}$and $\mathrm{CD}^{+} \mathrm{T}$ cell allostimulatory capacity, as well as a potent indirect alloantigen presentation. We identified GM-CSF as one of the soluble factors produced by renal epithelial cells that, combined with TLR9 ligation, induced this functional capacity. Our data suggest that pDCs present in the rejecting allograft can contribute to alloimmunity and can potentially act as important orchestrators in the manifestation of acute and chronic rejection.
\end{abstract}

\title{
Keywords
}

apoptosis, cytomegalovirus, acute rejection, chronic allograft nephropathy, endothelium, lymphocytes 


\section{Introduction}

Plasmacytoid dendritic cells (pDCs) were first classified as dendritic cells (DCs) in the 1990s ${ }^{1}$, and have since been shown to be capable of acting as antigen presenting cells (APCs) ${ }^{1-3}$. pDCs selectively express the endosomal Toll-like receptor (TLR)7 and TLR9 ${ }^{4}$, which allows for the detection of viral and bacterial single stranded RNA and hypomethylated DNA respectively ${ }^{5,6}$. Ligation of these TLRs leads to the production of vast amounts of interferon alpha (IFN $\alpha$ ), and pDCs are hence referred to as the type I IFN producing cells. These abilities stage pDCs as APCs instrumental for the clearance of infections ${ }^{7-9}$. However, pDCs can also express proteins involved in the induction of T cell tolerance, e.g. Indoleamine-pyrrole 2,3-dioxygenase (IDO), Delta-Like-4 , PD-L1 and Granzyme B ${ }^{10-13}$.

APCs are key in the manifestation of both acute and chronic rejection in solid organ transplantation. Passenger APCs of donor origin can induce allo-reactive T cell activation via the direct antigen $(\mathrm{Ag})$ recognition pathway early after transplantation, whereas APCs of the recipient are involved in chronic rejection by mediating indirect Ag presentation ${ }^{14}$. Although in theory any activated passenger APC can induce direct allo-reactivity, the induction of $C D 4^{+}$ $\mathrm{T}$ cells with indirect specificity requires the phagocytosis of donor cell-derived $\mathrm{Ag}$, e.g. apoptotic cells, and subsequent presentation of the acquired Ag in the context of MHC class II by mature APCs.

APC maturation can be induced by infections, via the recognition of pathogenassociated molecular patterns by e.g. TLRs. Transplanted patients are susceptible to opportunistic infections due to their immune suppressed state, and especially infection with polyomaviruses (BK virus) and cytomegalovirus (CMV) causes morbidity and mortality ${ }^{15}$. Interestingly, multiple studies have suggested a relation between infection (especially with $\mathrm{CMV}$ ) and allograft rejection, despite remaining inconclusive due to the complexity of this interplay ${ }^{16}$.

Our laboratory has previously shown a strong influx of pDCs in the tubulointerstitium of renal biopsies taken at time of rejection, as compared to those taken at time of transplantation ${ }^{17}$. As such, pDCs could be bridging infection and allograft loss ${ }^{18}$. Despite these observations, the role of pDCs in the manifestation of solid organ rejection remains elusive. Experimental transplantation models have demonstrated that pDCs are capable of allo-Ag presentation ${ }^{18-20}$. Moreover, an IFN $\alpha$ signature was shown clinically during chronic antibody-mediated rejection, which coincided with an increased number of pDCs within the graft ${ }^{21}$. In addition, human pDCs are able to ingest antigen, e.g. virions, synthetic long peptides, and exosomes ${ }^{22-24}$. However, data on the ability of pDCs to ingest 
apoptotic cells (ACs) remains controversial, and hence their potential role in mediating chronic rejection. It has been suggested that pDCs can only phagocytose infected ACs ${ }^{25,26}$, while other studies have demonstrated pDCs to have a very limited capacity to ingest ACs ${ }^{27}$.

Since we observed an influx of pDCs in renal tissue at time of rejection, we set out to assess whether human pDC biology, and especially phagocytosis, is affected by their presence in renal tissue, and whether viral infection alters this interaction. Here we demonstrate that TLR9 ligation of $\mathrm{pDC}$ in the presence of tissue cell-produced soluble factors, including GM-CSF, initiates pDC's phagocytic capacity and strongly enhances their function. Our data position pDCs as APCs capable of mounting allogeneic immune responses via the indirect Ag presentation pathway, following activation by TLR9 ligation in the presence of tissue cell-produced soluble factors. 


\section{Results}

CpG-mediated PDC phenotypic maturation and IFN $\alpha$ production are enhanced following co-culture with renal epithelial cells

Recently, we demonstrated a strong influx of pDCs in the tubulointerstitium of biopsies of patients with acute allograft rejection ${ }^{17}$. Since pDCs were present in close proximity of the tubules, we investigated whether human kidney proximal tubular epithelial (HK2) cells could affect phenotypic maturation and IFNa production of purified blood-derived human pDCs.

In absence of any activating stimuli, culturing pDCs alone, or in the presence of viable HK2 cells did not lead to clear alterations in phenotypic maturation (Fig. 1a) nor IFNa production (Fig. 1b). Mimicking infection by the addition of the synthetic TLR9 ligand CpG led to the upregulation of the co-stimulatory molecule CD86, as well as the co-inhibitory molecule PD-L1 (Fig. 1C). As expected, pDC activation by CpG led to a robust IFN $\alpha$ production (Fig. 1d). Interestingly, CpG activation of pDCs in the presence of viable HK2 cells led to a significant increase in the expression of the costimulatory molecules CD86 and CD83, which coincided with a markedly reduced increase in the expression of the co-inhibitory molecule PD-L1 (Fig. 1c, black bars), compared to pDCs activated alone (Fig. 1c, white bars). In addition, the production of IFN $\alpha$ was significantly increased when pDCs were activated in the presence of HK2 cells (Fig. 1d), whereas CpG treatment of HK2 cells alone did not result in measurable IFN $\alpha$ production (data not shown). These data demonstrate that the presence of HK2 cells enhances CPG-induced PDC activation.

To study the effect of genuine viral infection, we setup a model using a GFPexpressing CMV strain. To this end, HK2 cells were infected with a varying multiplicity of infection (MOI). The infection efficiencies were CMV-dose-dependent, and the infection peak was observed 72 hours post infection (Fig. 1e) with a mean efficiency of $43 \%$ (range $14 \%-75 \%)$.

PDC maturation and cytokine production is increased by conditioned medium of CMV-infected HK2 cells

To address the effect of soluble factors produced by HK2 cell on pDCs, we harvested conditioned medium from non-infected HK2 (HCM) and CMV-infected HK2 cells (HCCM), and assessed the effect on pDC phenotypic maturation using flow cytometry (Fig. 2a). Quantification of the phenotypic analysis revealed that the expression of most activation markers remained low when pDCs were cultured in the presence of HCM for 48 hours. 
However, following culture with HCCM the expression of CD40 ( $p=0.058), \operatorname{CD86}(p=0.005)$ and MHC II ( $p=0.007)$ significantly increased (Fig 2b).

Analysis of the cytokine production showed that most of the tested cytokines were unaffected or affected to a minor extent by culturing pDCs in the presence of HCM or HCCM (Fig. $2 \mathrm{c}$ and data not shown), as compared to untreated pDCs. However, the production of IL-12p70 and IFN $\alpha$ by pDCs was significantly increased by HCCM, as compared to culture conditions without any stimulation or HCM (Fig. 2c). The induction of pDC maturation could (in part) be the result of remaining CMV virions within HCCM. Indeed, CMV DNA could be detected in HCCM by PCR, as opposed to HCM, albeit at lower levels compared to the original infection medium (Fig. 2d). Although viral DNA was detected in HCCM, we were unable to directly infect pDCs with CMV, showing that the increased activation of pDCs by HCCM cannot be caused by PDC infection (data not shown). These data demonstrate that pDCs become phenotypically mature and produce pro-inflammatory cytokines following culture in the presence of conditioned medium of virally infected HK2 cells.

\section{Activation by HCCM initiates pDC phagocytic capacity and subsequent maturation}

pDCs are known to be able to act as APCs in transplantation models ${ }^{18-20}$, which suggests they harbor the capacity to ingest donor antigen. Despite discrepancies in literature on the ability of pDCs to phagocytose ACs, consensus is that pDCs are capable of ingesting particulate antigen, but have a very limited capacity to ingest $A C^{27}$. To investigate the effect of the conditioned media on pDC phagocytosis, we induced apoptosis by low-dose $\mathrm{H}_{2} \mathrm{O}_{2}$ in HK2 cells. pDCs were co-cultured overnight with fluorescently labeled AC in presence or absence of $\mathrm{HCM}$ or HCCM. The percentage of $\left(\mathrm{BDCA}-2^{\mathrm{POS}} / \mathrm{CD} 123^{\mathrm{POS}}\right)$ pDCs capable of phagocytosing $A C$ was determined by analyzing the percentage of pDCs positive for CFSE using flow cytometry (Fig. 3a). In accordance with literature, pDCs showed a very limited capacity to ingest AC without stimulation (Fig. 3b). However, a robust increase in the phagocytic capacity of pDCs was achieved when AC were added in the presence of HCCM. In contrast, phagocytosis was not significantly increased in the presence of $\mathrm{HCM}$, free CMV, or when using $A C$ derived from CMV-infected HK2 cells (Fig 3b; HCCM, HCM, CMV, and ACMV respectively). The phagocytosis induced by $\mathrm{HCCM}$ was an active process, since $A C$ uptake was enhanced only when pDCs were cultured at $37{ }^{\circ} \mathrm{C}$ and not at $4{ }^{\circ} \mathrm{C}$ (Fig. 3C). To further confirm AC internalization, confocal microscopy was performed (Fig. 3d) and HLA-A2 negative pDCs were cultured overnight with CFSE-labeled HLA-A2 positive AC (Fig. 3e upper histogram), after which CFSE and HLA-A2 positivity was determined on pDCs (Fig. 3e lower 
plot). Only a minor portion $(<1 \%)$ of pDCs were both CFSE and HLA-A2 positive, demonstrating that the larger portion of CFSE positivity (being AC) was internalized.

Since DC maturation can be negatively affected by phagocytosis of $A C{ }^{28,29}$, we analyzed pDC phenotype by flow cytometry following incubation with $\mathrm{AC}$, and subsequently discriminating between pDCs which had (+) or had not (-) ingested AC (Fig. 3f). Whereas the expression of MHC class I and II was unaffected, pDCs which had ingested AC in fact showed an increased expression of CD40, CD80, CD83, CD86 and CCR7 (Fig.3f).

\section{HCCM-induced PDC phagocytosis is independent of the type of cell death or apoptotic cell source, and enhances indirect antigen presentation}

These data demonstrate that pDCs acquire the ability to become efficient phagocytes upon priming with soluble factors from CMV-infected HK2 cells. To address whether the type of apoptosis induction or AC source could affect the capacity of pDCs to ingest cell-derived material, we induced apoptosis in HK2 cells by means of UV- irradiation, or in endothelial cells (ECRF) by low-dose $\mathrm{H}_{2} \mathrm{O}_{2}$. AC derived from both UV-irradiated $\mathrm{HK} 2$ cells and from $\mathrm{H}_{2} \mathrm{O}_{2}$ treated ECRF were efficiently phagocytosed by pDCs cultured in the presence of HCCM (Fig. 4a). In addition, HCCM-activated pDCs were able to ingest necrotic cells, a process which was hampered when pDCs were kept at $4^{\circ} \mathrm{C}$ (Fig. 4b).

We next assessed whether the phagocytic capacity could also be induced by conditioned medium from other infected cell types. Whereas conditioned medium of noninfected endothelial cells (ECM) could not, conditioned medium from CMV-infected endothelial cells (ECCM) induced the phagocytic capacity of pDCs (Fig. 4c). Likewise, conditioned medium from non-infected primary proximal tubular epithelial cells (PTEC) had a minor effect on phagocytosis, whereas conditioned medium of CMV-infected PTEC increased the phagocytosis significantly (Fig. $4 d, p \leq 0.05$ ).

Finally, using a $C D 4^{+} \mathrm{T}$ cell clone with indirect specificity ( $\mathrm{T}$ cell clone 4.44 , which recognizes the aa101-114 epitope of HLA-A*201 in the context of HLA-DRB $1 * 0101{ }^{30}$ ), we investigated the indirect alloantigen presenting capacity of pDCs. Different concentrations of the HLA-A*201 synthetic long peptide (SLP, aa91-120) were incubated with HLA-A2 ${ }^{\text {neg }}$, HLADR0101 ${ }^{\text {pos }}$ pDCs in the absence or presence of conditioned medium. After $24 \mathrm{hrs}$ co-culture with $\mathrm{T}$ cell clone 4.44 , the IFNy production was determined as a measure for $\mathrm{T}$ cell activation resulting from indirect $\mathrm{Ag}$ presentation. HCCM-treated $\mathrm{pDC}$ induced a more pronounced indirect $\mathrm{Ag}$ presentation, both in the magnitude of the IFNY response and the SLP concentration required for the production of IFNY (Fig 4d). 


\section{$\mathrm{CD4}^{+}$and $C D 8^{+} \mathrm{T}$ cell proliferation and $T$ cell cytokine production is increased by}

HCCM-activated pDCs

Next, we determined whether the maturation of pDCs induced by HCCM resulted in an altered ability to induce $T$ cell activation. To this end we performed a mixed leucocyte reaction (MLR). pDCs were activated overnight, washed, and subsequently co-cultured with CFSE-labeled allogeneic peripheral blood lymphocytes at different ratios. After 5 days, $\mathrm{T}$ cell proliferation was assessed using flow cytometry, by determining the CFSE dilution of CD4 ${ }^{+}$ and $\mathrm{CD}^{+} \mathrm{T}$ cells, by their respective gating within the viable (7AAD ${ }^{\mathrm{NEG}}$ ) $\mathrm{T}$ cell $\left(\mathrm{CD} 3^{\mathrm{POS}}\right.$ ) population (Fig. 5a). As expected, culturing PBL with different ratios of pDCs resulted in a dose-dependent induction of proliferation (Fig. 5b). Quantification of the proliferation induced by non-activated pDCs showed a limited $\mathrm{CD} 4^{+}$and $\mathrm{CD} 8^{+} \mathrm{T}$ cell proliferation (Fig. 5c). Despite the lack of phenotypic maturation and minimal cytokine production (Fig. 2a-c), pDCs cultured overnight with $\mathrm{HCM}$ induced T cell proliferation to some degree (Fig. $5 b-c$ ). Interestingly, whereas $\mathrm{CD}^{+} \mathrm{T}$ cell proliferation was increased to a minor extent, overnight activation of PDCs with HCCM significantly enhanced their capacity to induce $\mathrm{CD} 4^{+} \mathrm{T}$ cell proliferation (Fig 5c).

Subsequent analysis of $T$ cell cytokines showed that $T$ helper cell $\left(T_{H}\right)$ cytokines were produced to some degree, with the exception of the $T_{H} 2$ cytokine IL-4 (Fig. $5 \mathrm{~d}$ ). The $T_{H} 17$ cytokine IL-17 was produced at comparable levels, irrespective of the type of stimulus. In contrast, HCCM-activated PDCs strongly increased the production of $T_{H} 1$ cytokine IFN $\gamma$, compared to the response induced by non- or HCM-activated pDCs (Fig. $5 \mathrm{~d}$ ). The activation with HCCM also led to increased production IL-10, albeit at lower absolute levels compared to HCM.

These data demonstrate that pDCs activated with HCCM induce significant $\mathrm{CD} 4^{+}$and $\mathrm{CD}^{+} \mathrm{T}$ cell proliferation, as well as a $\mathrm{T}_{\mathrm{H}} 1$ skewed cytokine profile.

Infection of HK2 cells is not a prerequisite for the induction of phagocytosis, but the combined activation by tissue-produced factors and TLR9 ligation is.

To gain insight in the soluble factors produced, we analyzed HCM and HCCM by ELISA (IL-3 and IFN $\alpha$ ) and Luminex (Fig. 6a). Most of the tested cytokines/chemokines were not produced by HK2 cells, or were produced at low levels (Fig 6a, white bars), irrespective of CMV infection (Fig 6a, black bars). In contrast, HK2 cells produced large amounts of IL-6 and IL-8, and substantial levels of GM-CSF, G-CSF, MCP-1, TNF $\alpha$ and IFNY (Fig. 6a, white bars), 
which were marginally increased following infection (Fig. 6a, black bars). Importantly, we were unable to detect the PDC survival factors IL-3 and IFN $\alpha$ in either conditioned medium.

Since we observed a minor difference in the analyzed factors between HCM and HCCM, we next determined whether viral infection of HK2 was indeed required for the induction of phagocytosis. pDCs that were cultured with AC from CMV-infected cells ( $A C^{\mathrm{CMV}}$ ) or with non-infected $\mathrm{AC}$ in the presence of $\mathrm{CpG}$, or $\mathrm{HCM}$ did not significantly increase their phagocytosis (Fig. 6b). In contrast, HCCM clearly enhanced phagocytosis, which could be slightly increased by the addition of $\mathrm{AC}^{\mathrm{CMV}}$ or $\mathrm{CpG}$. Importantly, combining $\mathrm{HCM}$ with either $A C^{C M V}$ or with $C p G$ also led to a strong increase in phagocytosis, mimicking the effect observed with HCCM. In addition to the HK2 cell line, supplementation of CpG to conditioned medium of primary PTECs also led to a marked increase in phagocytosis (Fig. 6c). These data show that the induction of phagocytosis is the result of the combined activation of (a) soluble factor(s) and TLR9 ligand (i.e. $\mathrm{AC}^{\mathrm{CMV}}$ or $\mathrm{CpG}$ ).

To determine whether one of the detected cytokines was responsible for this phenotype, we supplemented recombinant cytokines to pDC culture medium, either alone or combined with CpG. None of the added cytokines affected phagocytosis, with the exception of GM-CSF, which significantly enhanced phagocytosis. Importantly, this effect was only observed when GM-CSF was combined with CpG (Fig. 6d). In order to confirm GMCSF was indeed the driving factor within the conditioned medium, we targeted GM-CSF using a neutralizing antibody. We first assessed the effectiveness of the antibody by neutralizing GM-CSF in culture medium which was supplemented with recombinant GM-CSF and CPG. Whereas the induction of phagocytosis was unaffected by the addition of an isotype antibody, targeting GM-CSF abrogated the increased phagocytosis induced by recombinant GM-CSF (Fig. 6e, recGM-CSF). Despite a consequent reduction, we could not fully abrogate phagocytosis by neutralizing GM-CSF in HCM (Fig. 6e, HCM), despite the fact that the mean GM-CSF concentration in HCM was $172 \mathrm{pg} / \mathrm{mL}$, compared to $500 \mathrm{pg} / \mathrm{mL}$ for recombinant GM-CSF. These data suggest that, next to GM-CSF, (an) additional factor(s) is present in the conditioned medium responsible for the observed phenotype. 


\section{Discussion}

Here, we demonstrate that TLR9 ligation of pDCs in the presence of conditioned medium of renal epithelial cells initiates their phagocytic capacity, and enhances antigen presentation and T cell activation. We identified GM-CSF as an active compound driving this process, although additional, currently unknown, factors are of importance within the conditioned medium. Importantly, the induction of phagocytosis was critically dependent on TLR9 ligation. Within the limitation of being an in vitro study, our data demonstrates that pDCs can aid in orchestrating allo-reactive immune responses. This is in line with our previous data, showing a strong influx of pDCs in the tubulointerstitium in renal biopsies at time of acute rejection ${ }^{17}$.

In the current study we used a GFP-tagged CMV strain to infect HK2 epithelial cells. Although CMV does not typically infect epithelial cells, whereas BK virus does, both viruses are able to ligate TLR9 ${ }^{31}$. The presence of the GFP tag in the used CMV strain allowed for the assessment of the infection efficiency. Our data demonstrates that pDCs become mature APC by activation with conditioned medium of CMV-infected HK2 cells (HCCM). We showed that viral DNA is present in HCCM, the presence of CMV cannot explain the induction of the phagocytic capacity in pDCs. We demonstrated that neither free virus, nor AC derived from CMV-infected cells, can induce phagocytosis as robust as HCCM. In line with this observation we showed that phagocytosis was only induced by TLR9 ligation in the presence of conditioned medium or GM-CSF.

The capacity to phagocytose AC can have important implications for the capacity of indirect antigen presentation, and, although this was not proven directly, we could indeed show an increase in indirect alloantigen presentation using a model system with an indirectly-reactive $\mathrm{CD}^{+} \mathrm{T}$ cell clone and a synthetic long peptide. Moreover, using an MLR we demonstrated that HCCM-activated pDCs displayed an increased capacity to induce $T$ cell priming and $T_{H} 1$ skewing. The production of IFN $\alpha$ and low levels of IL-12p70 by HCCM-activated pDCs could be driving the $T_{H} 1$ polarization we observed in the MLR. The latter is instrumental for the potent induction of antigen-specific $\mathrm{CD}^{+}$cytotoxic $\mathrm{T}$ cells, which are important for the clearance of virally-infected cells. Moreover, combined with the production of IFN $\alpha, \mathrm{CD}^{+} \mathrm{T}$ cell activation is the driving force for antibody production by $\mathrm{B}$ cells ${ }^{32}$, and this can explain the recently observed IFN $\alpha$ signature in PBMCs during chronic antibody-mediated rejection, which coincided with an increased number of pDCs within the graft ${ }^{21}$. These data further support the notion that, as the natural type I IFN producing cells, pDC could have an important role in mediating renal allograft rejection. 
Whether GM-CSF and other soluble factors are secreted at higher levels following CMV infection in vivo remains to be determined. It is established that RIG-I-like receptors, as well as TLR7 and TLR9, are capable of detecting (cytosolic/endosomal) nucleic acids ${ }^{33,34}$, and epithelial cells should therefore be able to detect early CMV infection ${ }^{35}$. Following viral sensing, infected cells produce IFNs ${ }^{36}$ which could coincide with the secretion of aditional soluble factors. Recruited pDCs can subsequently become primed for phagocytosis and initiate an anti-viral response. In the context of transplantation, this can directly impact allogeneic immunity by potentially activating $T$ cells with indirect specificity. To determine the in vivo relevance of pDCs in inducing alloimmunity, future studies should include the examination of the in situ activation status of, and IFN $\alpha$ production by, pDCs in rejection biopsies compared to biopsies without rejection, and whether activated pDCs can be detected in close proximity of infected cells.

With the identification of GM-CSF as one of the active compounds within HCM, it is of interest to note that the prototypic PDC cytokine receptor IL-3 receptor- $\alpha$ (CD123), together with IL-5 receptor- $\alpha$ (CD125), belongs to the same receptor family as the GM-CSF receptor- $\alpha(C D 116){ }^{37}$. Although IL-3 is known to act as a potent survival factor for pDCs, its expression within peripheral tissues is restricted ${ }^{38,39}$. In contrast, GM-CSF is produced in (inflamed) tissues, as well as by activated leukocytes ${ }^{40,41}$, suggesting GM-CSF is of importance for $\mathrm{pDC}$ function in peripheral tissues. The data presented here, together with the central role of GM-CSF as a potentiating cytokine for multiple cells of the myeloid compartment could position GM-CSF as a key player in mediating alloimmunity. Being essential in de initiation of allogeneic immune responses ${ }^{42}$, inhibiting APC activation by targeting GM-CSF could therefore be instrumental.

In conclusion, we have shown that renal epithelial cells produce GM-CSF and additional, currently unknown, soluble factors, which induce pDC maturation, proinflammatory cytokine production and potently induce their phagocytic capacity upon TLR9 ligation by $\mathrm{CMV}$-infected AC or CpG. HCCM-activated pDCs subsequently increase indirect Ag presentation and are capable of robustly activating $\mathrm{CD} 4^{+}$and $\mathrm{CD} 8^{+} \mathrm{T}$ cells. Our data therefore point to pDCs as potent APC capable of orchestrating allogeneic immune responses, and could be key players in the induction of chronic rejection. 


\section{Methods}

\section{pDC isolation and cell lines}

Ficoll density separation was used to isolate peripheral blood mononuclear cells (PBMC) from buffy coats of healthy individuals, which were obtained after informed consent (Sanquin, The Netherlands). pDCs were purified from freshly isolated PBMC by negative MACS selection, using the Plasmacytoid dendritic cell isolation kit II following manufacturers protocol (Miltenyi Biotec, The Netherlands). pDC purity was assessed by flow cytometry (LSRII, BD Biosciences) using BDCA-2 FITC and CD123 PE (BD Biosciences), which exceeded $90 \%$ in all cases.

Human kidney proximal tubular epithelial (HK2) cells were cultured in DMEM HAM F12 (Lonza, The Netherlands), supplemented with Tri-iodo thyronine (Sigma), ITS-X (insulintransferrin-selenium-ethanolamine, Gibco), Epidermal growth factor (Sigma), Hydrocortison (Sigma) and $100 \mathrm{U} / \mathrm{mL}$ Penicillin-Streptomycin (Gibco).

Human ECRF endothelial cells were cultured in medium 199 (Gibco), supplemented with 20\% FCS, $10 \mathrm{U} / \mathrm{mL}$ heparin (LEO Pharma, Canada), 1 tube $(25 \mathrm{mg}$ ) bovine pituitary gland extract (Life Technologies) and $100 \mathrm{U} / \mathrm{mL}$ Penicillin-Streptomycin (Gibco).

All cell lines were regularly tested negative for Mycoplasm infection.

\section{Induction of cell death}

Apoptosis was induced by harvesting HK2 or ECRF cells and subsequent culture in the presence of $8 \mu \mathrm{M} \mathrm{H}_{2} \mathrm{O}_{2}$. Alternatively, HK2 cell apoptosis was induced by $400 \mathrm{~J} / \mathrm{m}^{2} \mathrm{UV}$ irradiation. After 48 hours $>95 \%$ of the apoptotic HK2 cells were secondary necrotic (Annexin $V$ and 7AAD positive), which was assessed by flow cytometric analysis of Annexin A5-FITC (VPS Diagnostics, The Netherlands) and 7AAD (BD Biosciences) staining. Necrosis was induced by heating the cells to $56^{\circ} \mathrm{C}$ for $1 \mathrm{hr}$, after which all cells were 7AAD positive. The necrotic cells were washed and used directly.

\section{Cytomegalovirus infection and conditioned medium}

HK2 or ECRF cells were cultured overnight to $80 \%$ confluence, after which the culture medium was removed and the cells were infected with human CMV TB40-GFP ${ }^{43}$ at a MOI of $100-200$. To enhance infection, cells were centrifuged at $37^{\circ} \mathrm{C}$ for 1 hour at $500 \times \mathrm{g}$. After $48-72$ hours of culture, the conditioned medium was harvested, centrifuged at $3500 \times \mathrm{g}$, and the supernatant was stored at $-20{ }^{\circ} \mathrm{C}$ for further use. The infection efficiency was assessed by determining the GFP expression using flow cytometry (LSR-II, BD Biosciences). 


\section{Cytokine analysis}

pDC and HK2 culture supernatants were analyzed for IFN $\alpha$ (pan IFN $\alpha$, Mabtech, detection limit $30 \mathrm{pg} / \mathrm{mL}$ ) or IL-3 (Peprotech, detection limit $30 \mathrm{pg} / \mathrm{mL}$ ) production using a sandwich ELISA. All other cytokines and chemokines were determined using Luminex (Bio-rad, detection limit $<10 \mathrm{pg} / \mathrm{mL}$ ).

\section{Phagocytosis assay}

AC were isolated, labeled with $4 \mu \mathrm{M}$ CFSE (Thermo Fisher), and washed once with serumcontaining medium. The CFSE labelling was left to settle for at least one hours, after which the $A C$ were washed and used for phagocytosis experiments. Fresh pDCs were cultured with $A C$ at a 1:1 ratio and the number of pDCs positive for CFSE was determined using flow cytometry, after overnight culture at $4{ }^{\circ} \mathrm{C}$ versus $37^{\circ} \mathrm{C}$.

\section{Indirect antigen presentation}

Indirect $\mathrm{Ag}$ presentation was assessed using $\mathrm{CD} 4^{+} \mathrm{T}$ cell clone 4.44 , which recognizes the aa101-114 epitope of HLA-A*201 in the context of HLA-DRB1*0101 ${ }^{44}$. To this end, $2 * 10^{4}$ pDCs (HLA-A2 ${ }^{\text {neg }}$, HLA-DRB1*0101 ${ }^{\text {pos }}$ ) were loaded with an aa91-120 HLA-A*201 synthetic long peptide (SLP) in the presence of different stimuli. After overnight loading and activation, $\mathrm{pDCs}$ were washed and $\mathrm{CD}^{+} \mathrm{T}$ cell clone 4.44 was added at a 1:1 ratio. Supernatants were harvested after 24 hours and IFN $y$ production was analyzed by ELISA (eBioscience).

\section{Mixed leukocyte reaction}

Peripheral blood lymphocytes (PBL) were isolated by CD14 MACS depletion (Miltenyi Biotec) of fresh PBMC, and were labeled with $1 \mu \mathrm{M}$ CFSE (Thermo Fisher). Allogeneic pDCs were activated overnight, washed and subsequently co-cultured with $1 * 10^{5} \mathrm{PBL}$ at $\mathrm{pDC}: \mathrm{PBL}$ ratio's ranging from 1:10 to 1:40. After 5 days, culture supernatants were isolated and stored at $-20^{\circ} \mathrm{C}$ for Luminex analysis (Bio-rad). T cell proliferation was assessed by analyzing the CFSE dilution on viable $\mathrm{CD} 3 / 4^{+} \mathrm{CD} 3 / 8^{+} \mathrm{T}$ cells by flow cytometry (LSR-II, BD Biosciences).

\section{Cytokine supplementation and neutralization}

Recombinant cytokines alone, or combined with $\mathrm{CpG}$, were supplemented to pDC cultures at the maximum concentrations detected in the conditioned medium, i.e. IL-6 $(10 \mathrm{ng} / \mathrm{mL}, \mathrm{R} \& \mathrm{D}$ 
systems), IL-8 (20 ng/mL, eBioscience), GM-CSF (800 pg/mL, Miltenyi Biotec), G-CSF (12 $\mathrm{ng} / \mathrm{mL}, \mathrm{R} \& \mathrm{D}$ systems), MCP-1 (300 pg/mL, R\&D systems), TNF $\alpha$ (100 pg/mL, Biolegend) and IFNY (75 pg/mL, Peprotech). In order to neutralize GM-CSF, a-GM-CSF (clone \# 3209, R\&D systems) was added to $\mathrm{HCM}$ or $500 \mathrm{pg} / \mathrm{mL}$ recombinant GM-CSF (Peprotech) at $5 \mu \mathrm{g} / \mathrm{mL} 1$ hour prior to use. After overnight culture in the presence of CFSE-labelled AC, phagocytosis was analysed using flow cytometry.

\section{Statistical analysis}

Statistical analysis was performed using a paired Student's $t$-test, or an one-way ANOVA with Tukey's Multiple-Comparison Test. P-values of $\leq 0.05$ were considered significant.

\section{Disclosures}

The authors have no conflicts of interest to disclose.

\section{References}

1. Grouard G, Rissoan MC, Filgueira L et al. The enigmatic plasmacytoid T cells develop into dendritic cells with interleukin (IL)-3 and CD40-ligand. J. Exp. Med. 1997; 185: 1101-11.

2. Mouriès J, Moron G, Schlecht $\mathrm{G}$ et al. Plasmacytoid dendritic cells efficiently cross-prime naive T cells in vivo after TLR activation. Blood 2008; 112: 3713-22.

3. Fonteneau J-F, Gilliet M, Larsson M et al. Activation of influenza virus-specific CD4+ and CD8+ T cells: a new role for plasmacytoid dendritic cells in adaptive immunity. Blood 2003; 101: 3520-6.

4. Heil F, Ahmad-Nejad P, Hemmi H et al. The Toll-like receptor 7 (TLR7)-specific stimulus loxoribine uncovers a strong relationship within the TLR7, 8 and 9 subfamily. Eur. J. Immunol. 2003; 33: 2987-97.

5. Hemmi H, Takeuchi O, Kawai T et al. A Toll-like receptor recognizes bacterial DNA. Nature 2000; 408: 740-5.

6. Diebold SS, Kaisho T, Hemmi H et al. Innate antiviral responses by means of TLR7mediated recognition of single-stranded RNA. Science 2004; 303: 1529-31.

7. Kadowaki N, Antonenko S, Lau JY-N et al. Natural Interferon / -Producing Cells Link Innate and Adaptive Immunity. J. Exp. Med. 2000; 192: 219-226.

8. Smit JJ, Rudd BD, Lukacs NW. Plasmacytoid dendritic cells inhibit pulmonary immunopathology and promote clearance of respiratory syncytial virus. J. Exp. Med. 2006;

203: 1153-9.

9. Wang H, Peters N, Schwarze J. Plasmacytoid Dendritic Cells Limit Viral Replication, Pulmonary Inflammation, and Airway Hyperresponsiveness in Respiratory Syncytial Virus Infection. J. Immunol. 2006; 177: 6263-6270. 
10. Munn DH, Sharma MD, Hou D et al. Expression of indoleamine 2,3-dioxygenase by plasmacytoid dendritic cells in tumor-draining lymph nodes. J. Clin. Invest. 2004; 114: 280 90.

11. Kassner N, Krueger M, Yagita $\mathrm{H}$ et al. Cutting edge: Plasmacytoid dendritic cells induce IL10 production in T cells via the Delta-like-4/Notch axis. J. Immunol. 2010; 184: 550-4.

12. Tokita D, Mazariegos G V, Zahorchak AF et al. High PD-L1/CD86 ratio on plasmacytoid dendritic cells correlates with elevated T-regulatory cells in liver transplant tolerance. Transplantation 2008; 85: 369-77.

13. Jahrsdörfer B, Vollmer A, Blackwell SE et al. Granzyme B produced by human plasmacytoid dendritic cells suppresses T-cell expansion. Blood 2010; 115: 1156-65.

14. Rogers NJ, Lechler RI. Allorecognition. Am. J. Transplant 2001; 1: 97-102.

15. Weikert BC, Blumberg EA. Viral infection after renal transplantation: surveillance and management. Clin. J. Am. Soc. Nephrol. 2008; 3 Suppl 2: S76-86.

16. Cainelli F, Vento $S$. Infections and solid organ transplant rejection: a cause-and-effect relationship? Lancet Infect. Dis. 2002; 2: 539-549.

17. Zuidwijk K, de Fijter JW, Mallat MJK et al. Increased influx of myeloid dendritic cells during acute rejection is associated with interstitial fibrosis and tubular atrophy and predicts poor outcome. Kidney Int. 2012; 81: 64-75.

18. Koyama M, Hashimoto D, Aoyama K et al. Plasmacytoid dendritic cells prime alloreactive T cells to mediate graft-versus-host disease as antigen-presenting cells. Blood 2009; 113: 2088-95.

19. Ochando JC, Homma C, Yang Y et al. Alloantigen-presenting plasmacytoid dendritic cells mediate tolerance to vascularized grafts. Nat. Immunol. 2006; 7: 652-62.

20. Björck P, Coates PTH, Wang $Z$ et al. Promotion of long-term heart allograft survival by combination of mobilized donor plasmacytoid dendritic cells and anti-CD154 monoclonal antibody. J. Heart Lung Transplant. 2005; 24: 1118-20.

21. Rascio F, Pontrelli P, Accetturo M et al. A type I Interferon signature characterizes chronic antibody-mediated rejection in kidney transplantation. J. Pathol. 2015.

22. Tel J, Lambeck AJA, Cruz LJ et al. Human plasmacytoid dendritic cells phagocytose, process, and present exogenous particulate antigen. J. Immunol. 2010; 184: 4276-83.

23. Bastos-Amador $\mathrm{P}$, Pérez-Cabezas $\mathrm{B}$, Izquierdo-Useros $\mathrm{N}$ et al. Capture of cell-derived microvesicles (exosomes and apoptotic bodies) by human plasmacytoid dendritic cells. J. Leukoc. Biol. 2012; 91: 751-8.

24. Villadangos J a, Young L. Antigen-presentation properties of plasmacytoid dendritic cells. Immunity 2008; 29: 352-61.

25. Hoeffel G, Ripoche A-C, Matheoud D et al. Antigen crosspresentation by human plasmacytoid dendritic cells. Immunity 2007; 27: 481-92.

26. Lui G, Manches O, Angel J et al. Plasmacytoid dendritic cells capture and cross-present viral antigens from influenza-virus exposed cells. PLoS One 2009; 4: e7111.

27. Dalgaard J, Beckstrøm KJ, Jahnsen FL et al. Differential capability for phagocytosis of apoptotic and necrotic leukemia cells by human peripheral blood dendritic cell subsets. J. Leukoc. Biol. 2005; 77: 689-98.

28. Liu K, Iyoda T, Saternus M et al. Immune Tolerance After Delivery of Dying Cells to 
Dendritic Cells In Situ. J. Exp. Med. 2002; 196: 1091-1097.

29. Sen $P$, Wallet MA, Yi Z et al. Apoptotic cells induce Mer tyrosine kinase-dependent blockade of NF-KB activation in dendritic cells. Blood 2007; 109: 653-660.

30. Breman E, van Miert PP, van der Steen DM et al. HLA monomers as a tool to monitor indirect allorecognition. Transplantation 2014; 97: 1119-27.

31. Xagorari A, Chlichlia K. Toll-like receptors and viruses: induction of innate antiviral immune responses. Open Microbiol. J. 2008; 2: 49-59.

32. McHeyzer-Williams LJ, McHeyzer-Williams MG. Antigen-specific memory B cell development. Annu. Rev. Immunol. 2005; 23: 487-513.

33. Pichlmair A, Schulz O, Tan CP et al. RIG-I-mediated antiviral responses to single-stranded RNA bearing 5'-phosphates. Science 2006; 314: 997-1001.

34. Yoneyama $\mathrm{M}$, Kikuchi $\mathrm{M}$, Matsumoto $\mathrm{K}$ et al. Shared and unique functions of the DExD/H-box helicases RIG-I, MDA5, and LGP2 in antiviral innate immunity. J. Immunol. 2005; 175: 2851-8.

35. Huang ES, Johnson RA. Human cytomegalovirus - no longer just a DNA virus. Nat. Med. 2000; 6: 863-4.

36. Rathinam VAK, Fitzgerald KA. Innate immune sensing of DNA viruses. Virology 2011; 411: 153-62.

37. Broughton SE, Dhagat U, Hercus TR et al. The GM-CSF/IL-3/IL-5 cytokine receptor family: from ligand recognition to initiation of signaling. Immunol. Rev. 2012; 250: 277-302.

38. Alexander WS. Cytokines in Hematopoiesis. Int. Rev. Immunol. 1998; 16: 651-682.

39. Nicola NA. Hemopoietic Cell Growth Factors and Their Receptors. Annu. Rev. Biochem. 1989; 58: 45-77.

40. Egea L, Hirata Y, Kagnoff MF. GM-CSF: a role in immune and inflammatory reactions in the intestine. Expert Rev. Gastroenterol. Hepatol. 2010; 4: 723-31.

41. Hamilton JA. Colony-stimulating factors in inflammation and autoimmunity. Nat. Rev. Immunol. 2008; 8: 533-544.

42. Alegre M-L, Lakkis FG, Morelli AE. Antigen Presentation in Transplantation. Trends Immunol. 2016; 37: 831-843.

43. Sinzger $\mathrm{C}$, Hahn G, Digel $\mathrm{M}$ et al. Cloning and sequencing of a highly productive, endotheliotropic virus strain derived from human cytomegalovirus TB40/E. J. Gen. Virol. 2008; 89: 359-68.

44. Amir AL, Hagedoorn RS, van Luxemburg-Heijs SAP et al. Identification of a coordinated CD8 and CD4 T cell response directed against mismatched HLA Class I causing severe acute graft-versus-host disease. Biol. Blood Marrow Transplant. 2012; 18: 210-9. 


\section{Acknowledgements}

We thank Els van Beelen for technical assistance in acquiring cytokine data by Luminex and Dr. Mariet Feltkamp for the determination of the CMV titers.

This research was funded by grant CP 09.04 (AlloVir) of the Dutch Kidney Foundation (Nierstichting Nederland). 
A

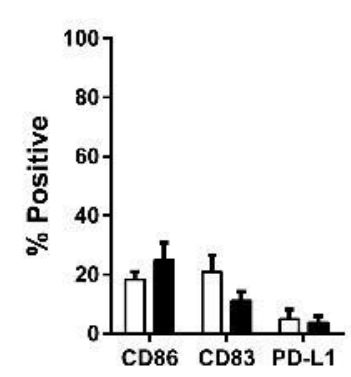

B

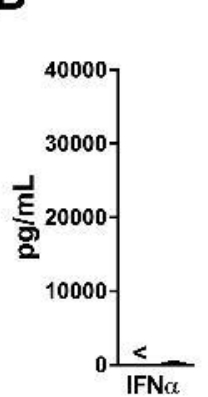

C

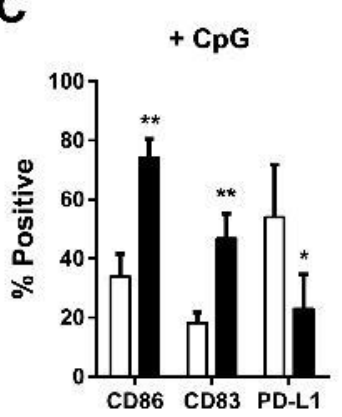

D

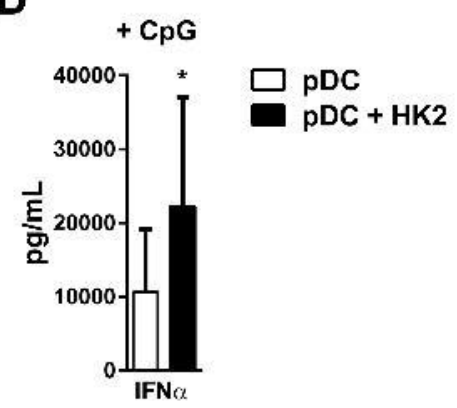

E

$48 \mathrm{hr}$

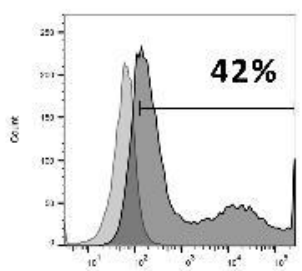

GFP

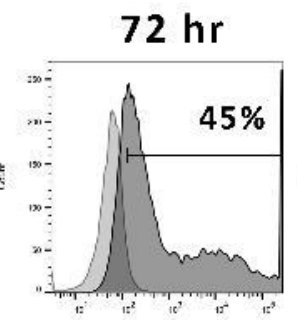

GFP

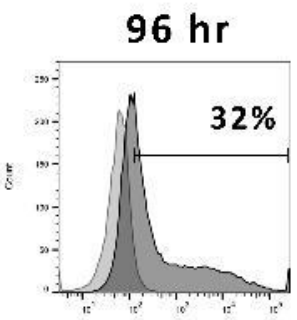

GFP

Figure 1: CpG-mediated $p D C$ phenotypic maturation and IFN $\alpha$ production are enhanced following co-culture with renal epithelial cells

(A) pDCs were cultured in the presence (black bars) or absence (white bars) of viable HK2 cells, after which cell surface expression of CD86, CD83 and PD-L1 was determined by flow cytometry and (B) IFN $\alpha$ production by ELISA. <: Cytokine levels $<10 \mathrm{pg} / \mathrm{mL}$. (C) pDCs were cultured in absence (white bars) or presence (black bars) of viable HK2 cells and subsequently activated with $\mathrm{CpG}$. The expression of cell surface markers was assessed by flow cytometry and (D) IFN $\alpha$ production by ELISA. $\mathrm{n}=6$; ${ }^{*} \mathrm{p}<0.05,{ }^{* *} \mathrm{p}=<0.01$; Student's $t$ test. (E) HK2 cells were infected with a GFP-expressing CMV strain (MOI 200), after which the infection efficiency was analyzed by flow cytometry at 48,72 and $96 \mathrm{hrs}$. 
A

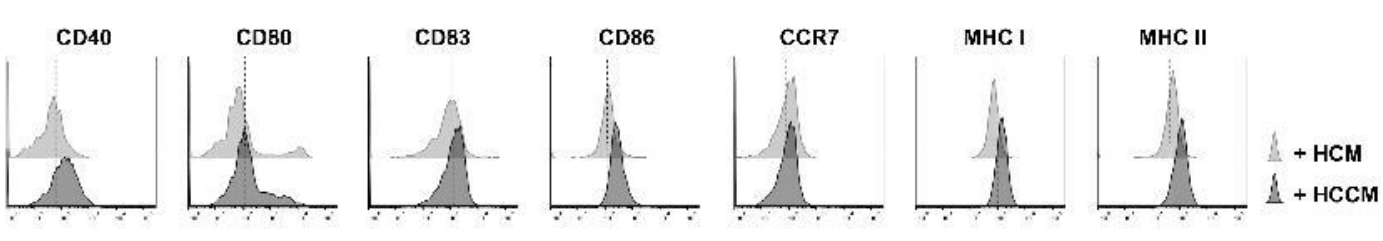

B

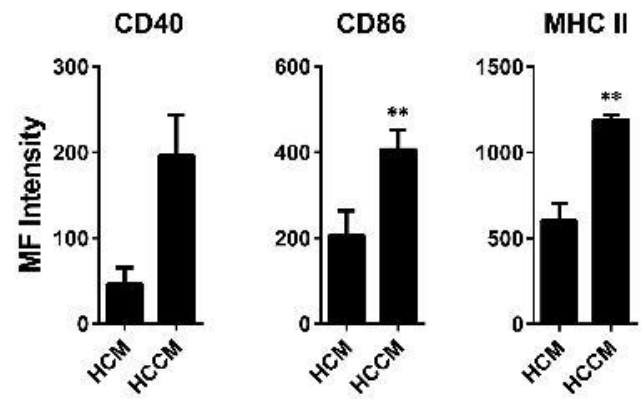

C

IL-10
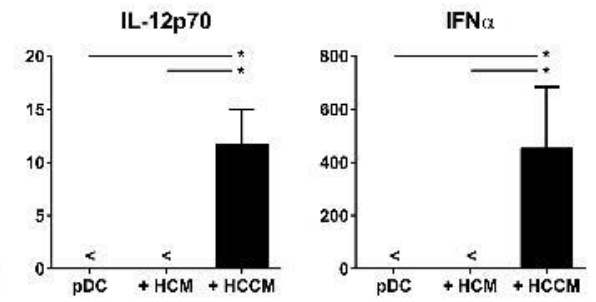

D

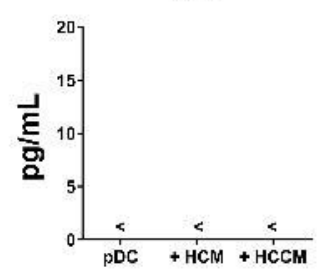

$\mathrm{PDC}+\mathrm{HCM}+\mathrm{HCCM}$

$\mathrm{PDC}+\mathrm{HCM}+\mathrm{HCCM}$

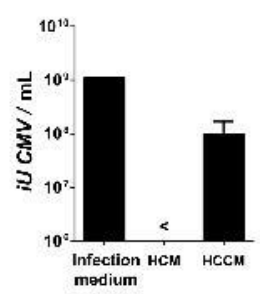

Figure 2: $p D C$ maturation and cytokine production is increased by conditioned medium of CMV-infected HK2 cells

(A) Representative flow cytometry plots of pDCs cultured with HCM (light grey) or HCCM (dark gray) for 48 hours. The line represents the mean value of unstimulated pDCs. (B) Quantification of pDC phenotype after culture with HCM or HCCM. $(n=3) ;{ }^{* *} p=<0.01$; Student's $t$-test. (C) 48 hour culture supernatants of pDCs cultured in absence or presence of HCM or HCCM, were analyzed by Luminex (IL-10 and IL-12p70, $n=3$ ) or ELISA (IFN $\alpha, n=6)$. $\mathrm{p}<0.05$; One-way ANOVA. (D) CMV titer of infection medium, HCM and HCCM, as determined by PCR. $(n=3) ;<$ : not detectable. 
A

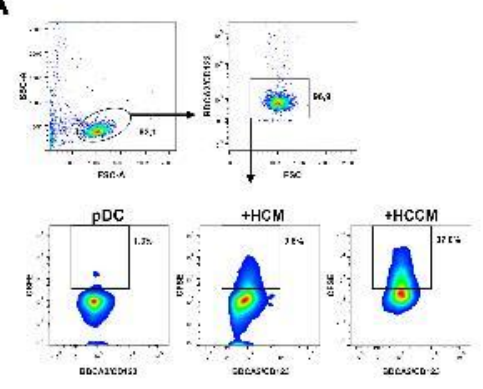

D

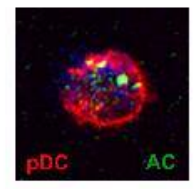

B

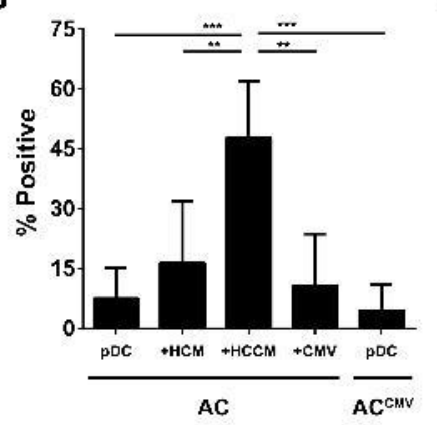

C

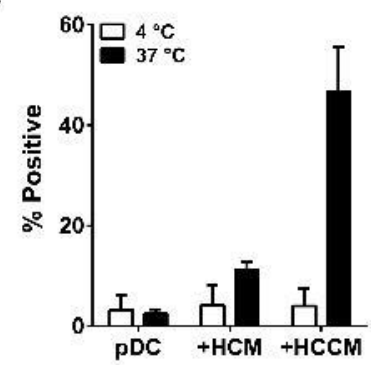

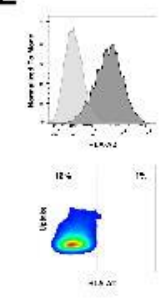

$\mathbf{F}$

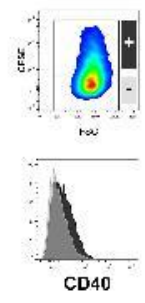

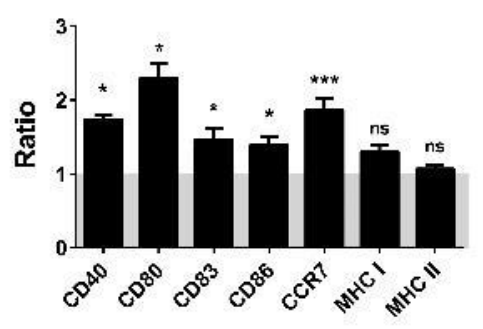

Figure 3: Activation by HCCM initiates $p D C$ phagocytic capacity and subsequent maturation

(A) pDCs were cultured overnight with CFSE-labeled AC in the presence or absence of conditioned media, after which BDCA-2 and CD123 positive pDCs were analyzed for CFSE positivity by flow cytometry. (B) Quantification of the phagocytosis of AC or CMV-infected $A C\left(A C^{C M V}\right)$ by $p D C s$, following overnight culture in the presence of different stimuli $(n=8$, $\mathrm{CMV}$ and $\mathrm{AC}^{\mathrm{CMV}} \mathrm{n}=3$ ). ${ }^{* *} \mathrm{p}=<0.01, * * * \mathrm{p}=<0.001$; One-way ANOVA. (C) Quantification of pDC phagocytosis of $A C$, following culture at $4{ }^{\circ} \mathrm{C}$ (white bars) or $37^{\circ} \mathrm{C}$ (black bars, $n=2$ ). (D) Confocal imaging of pDC were cultured overnight with CFSE-labeled AC (green) in the presence of HCCM and subsequently labeled with BDCA-2 and CD123 (red). (E) HLA-A2 negative pDCs (upper histogram, light grey graph) were cultured overnight with CFSElabeled, HLA-A2 positive AC (upper histogram, dark grey graph) in the presence of HCCM. Next, CFSE-positivity and HLA-A2 negativity was assessed by flow cytometry (lower plot) as a measure for internalization.

(E) Phenotypic analysis of pDCs by flow cytometry, following overnight culture with AC and HCCM. Expression ratio of pDCs which had (+) or had not (-) taken up AC. $n=3 ;{ }^{*} p=<0.05$, $* * * \mathrm{p}=<0.001 ;$ Student's $t$-test. 
A

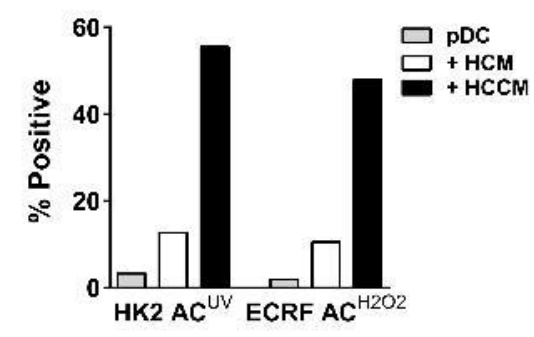

C
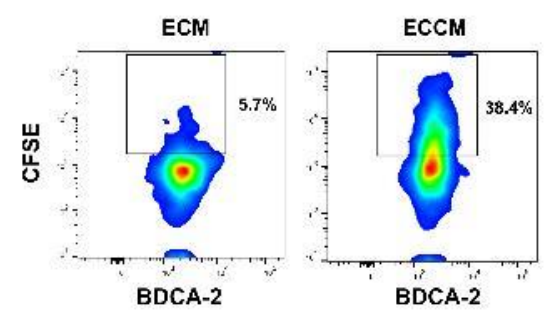

B

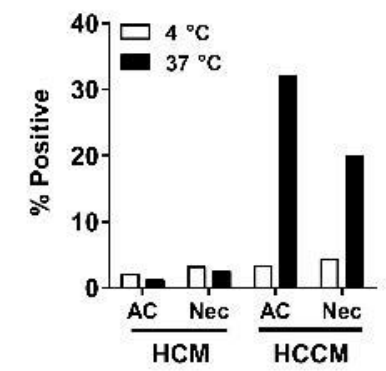

D

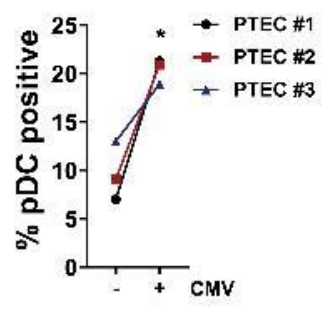

$\mathrm{E}$

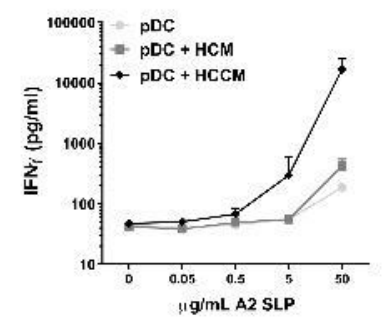

Figure 4: HCCM-induced $P D C$ phagocytosis is independent of the type of cell death or apoptotic cell source, and enhances indirect antigen presentation

(A) Phagocytosis of UV-irradiated HK2 epithelial AC or $\mathrm{H}_{2} \mathrm{O}_{2}$-induced ECRF endothelial AC, after overnight culture with no stimulus, HCM, or HCCM. $n=1$ (B) Phagocytosis of AC or necrotic cells $(\mathrm{Nec})$, after overnight culture in the presence of $\mathrm{HCM}$ or $\mathrm{HCCM}$, at $4{ }^{\circ} \mathrm{C}$ (white bars) versus $37^{\circ} \mathrm{C}$ (black bars). $\mathrm{n}=1$ (C) 72 -hour conditioned medium of non-infected (ECM) or CMV-infected endothelial ECRF cells (ECCM) was added to pDCs and AC. After overnight culture, phagocytosis was determined by flow cytometry. $n=1$ (D) Under similar conditions, pDC phagocytosis was assessed when cultured in the presence of conditioned medium derived from non-infected (- CMV) or infected (+ CMV) primary tubular epithelial cells (PTEC) $. \mathrm{n}=3 ;{ }^{*} \mathrm{p}=<0.05$; Student's $t$-test. (E) IFNy production by T cell clone 4.44, after 24 hour co-culture with pDCs that were loaded with an 30-mer HLA-A2 SLP in absence of any stimuli, or in the presence of HCM or HCCM $(n=2)$. 


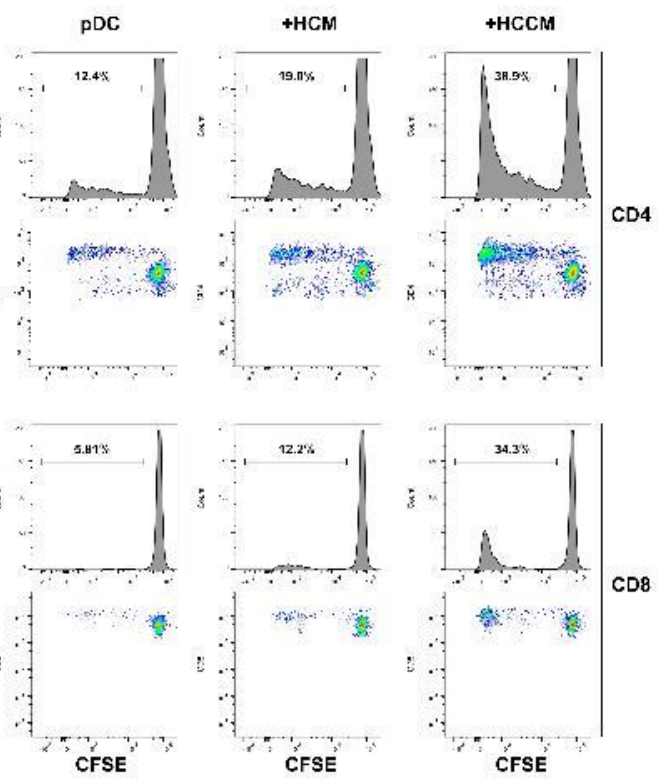

B

C
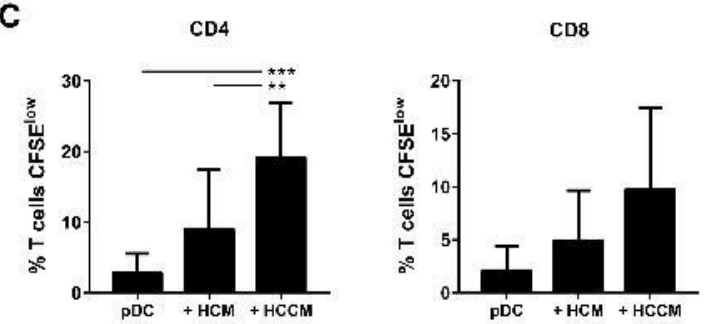

D
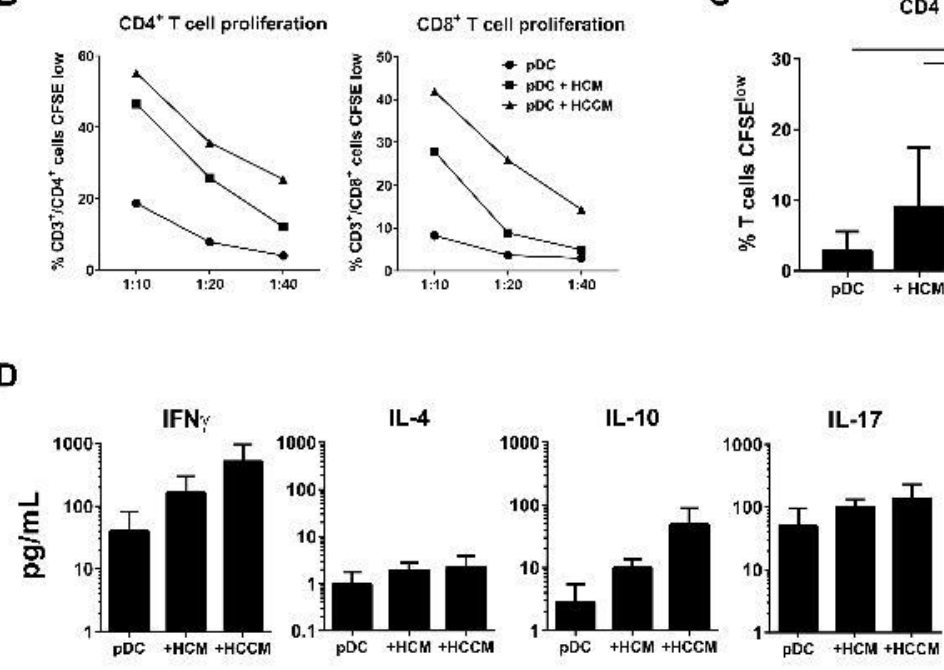

Figure 5: $C D 4^{+}$and $C D 8^{+} T$ cell proliferation and $T$ cell cytokine production is increased by HCCM-activated pDCs

pDCs were activated overnight with different stimuli, washed, and subsequently cultured with CFSE-labelled allogeneic peripheral blood lymphocytes, at PDC:PBL ratios of 1:10, 1:20 or 1:40. (A) After 5 days, CFSE dilution of viable $C D 4^{+}$and $C D 8^{+}$T cells (CD3 $\left.3^{+} / 7 A A D\right)$ was assessed by flow cytometry as a measure of $T$ cell proliferation (ratio 1:20). (B) Representative example of $\mathrm{T}$ cell proliferation following co-culture at different ratios with unstimulated pDCs, or pDCs cultured in the presence of HCM or HCCM. (C) Quantification of T cell proliferation at a pDC:PBL ratio of 1:20. $\mathrm{n}=8 ;{ }^{* *} \mathrm{p}=<0.01,{ }^{* * *} \mathrm{p}=<0.001$; One-way ANOVA. (D) After 5 days of co-culture, supernatants were analyzed by Luminex, for the production of IFNY, IL-4, IL-10 and IL-17. $n=2$ 
A

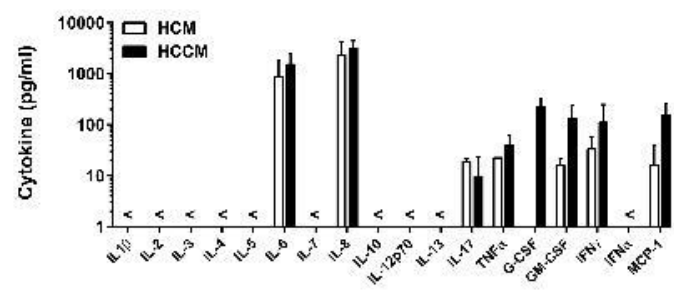

C

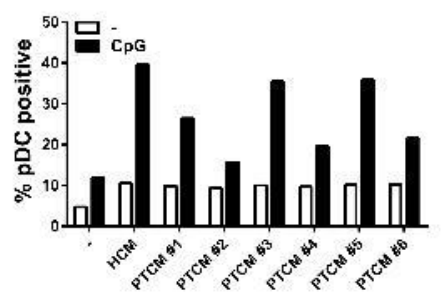

B

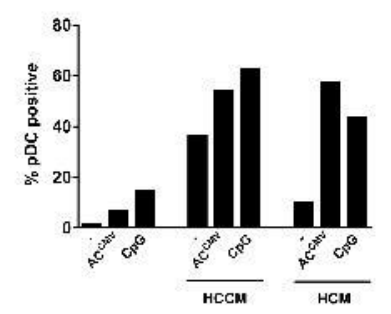

D

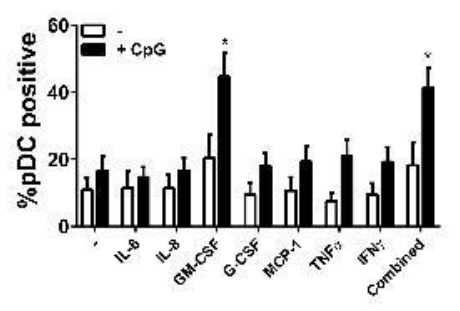

E

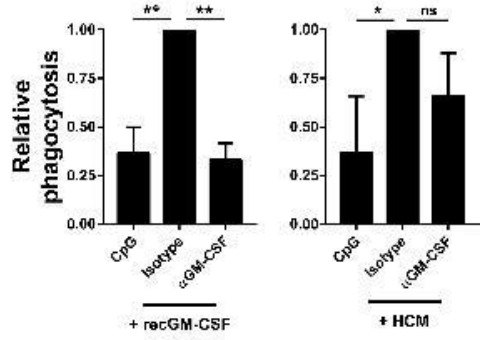

Figure 6: Infection of HK2 cells is not a prerequisite for the induction of phagocytosis, but the combined activation of soluble factors and a TLR9-ligand is.

(A) HK2 cells were cultured without infection (white bars) or infected with CMV (black bars), after which the produced soluble factors were assessed by ELISA (IL-3 and IFN $\alpha$ ) or Luminex after 72 hours. ( $n=2$ ); $<$ : Cytokine levels $<15 \mathrm{pg} / \mathrm{mL}$. (B) Phagocytosis induced by the single or combined activation of pDCs by TLR9-ligands ( $\mathrm{AC} \mathrm{CMV}^{\mathrm{C} V}$ or $\mathrm{CPG}$ ) or conditioned medium (HCCM or HCM). $n=1$ (C) The single or combined activation of pDCs by conditioned medium derived from multiple primary tubular epithelial cells (PTCM) and CpG. (D) Analysis of the effect of the cytokines detectable in conditioned medium alone (white bars) or combined with CpG (Black bars).(E) GM-CSF neutralization by either an isotype antibody, or GM-CSF neutralizing antibody, where the phagocytosis induced in the presence of an isotype antibody was set to 1. GM-CSF was neutralized in either $500 \mathrm{pg} / \mathrm{mL}$ recombinant GM-CSF (recGM-CSF, left graph), or HCM (mean GM-CSF concentration $172 \mathrm{pg} / \mathrm{mL}$; right graph). $\mathrm{n}=$ $4 ; * p=<0.05, * * p=<0.01$; One-way ANOVA. 